\title{
Stage IV Colon Cancer AJCC v7
}

National Cancer Institute

\section{Source}

National Cancer Institute. Stage IV Colon Cancer AJCC v7. NCI Thesaurus. Code C89999.

Stage IV includes: IVA: (Any T, Any N, M1a); IVB: (Any T, Any N, M1b). M1a: Metastasis

confined to one organ or site (e.g., liver, lung, ovary, nonregional node). M1 b:

Metastases in more than one organ/site or the peritoneum. (AJCC 7th ed.) 